\title{
Higher SARS-CoV-2 Seroprevalence in Workers with Lower Socioeconomic Status in Cape Town, South Africa.
}

\section{Jane Alexandra Shaw ( $\nabla$ janeshaw@sun.ac.za )}

Stellenbosch University - Tygerberg Campus: Stellenbosch University Faculty of Medicine and Health Sciences https://orcid.org/0000-0002-9893-3073

\section{Maynard Meiring}

Stellenbosch University - Tygerberg Campus: Stellenbosch University Faculty of Medicine and Health

Sciences

\section{Tracy Cummins}

Stellenbosch University - Tygerberg Campus: Stellenbosch University Faculty of Medicine and Health Sciences

\section{Novel Chegou}

Stellenbosch University Faculty of Medicine and Health Sciences

\section{Conita Claassen}

Stellenbosch University Faculty of Medicine and Health Sciences

\section{Nelita du Plessis}

Stellenbosch University - Tygerberg Campus: Stellenbosch University Faculty of Medicine and Health Sciences

\section{Marika Flinn}

Stellenbosch University - Tygerberg Campus: Stellenbosch University Faculty of Medicine and Health Sciences

\section{Andriette Hiemstra}

Stellenbosch University - Tygerberg Campus: Stellenbosch University Faculty of Medicine and Health

Sciences

\section{Leanie Kleynhans}

Stellenbosch University Faculty of Medicine and Health Sciences

\section{Vinzeigh Leukes}

Stellenbosch University - Tygerberg Campus: Stellenbosch University Faculty of Medicine and Health Sciences

\section{Andre G Loxton}

Stellenbosch University - Tygerberg Campus: Stellenbosch University Faculty of Medicine and Health Sciences

\section{Candice MacDonald}


Stellenbosch University - Tygerberg Campus: Stellenbosch University Faculty of Medicine and Health Sciences

\section{Nosipho Mtala}

Stellenbosch University - Tygerberg Campus: Stellenbosch University Faculty of Medicine and Health Sciences

\section{Helmuth Reuter}

Stellenbosch University - Tygerberg Campus: Stellenbosch University Faculty of Medicine and Health Sciences

\section{Donald Simon}

Stellenbosch University - Tygerberg Campus: Stellenbosch University Faculty of Medicine and Health Sciences

\section{Kim Stanley}

Stellenbosch University - Tygerberg Campus: Stellenbosch University Faculty of Medicine and Health

Sciences

\section{Gerard C Tromp}

Stellenbosch University - Tygerberg Campus: Stellenbosch University Faculty of Medicine and Health Sciences

\section{Wolfgang Preiser}

Stellenbosch University - Tygerberg Campus: Stellenbosch University Faculty of Medicine and Health Sciences

\section{Stephanus T Malherbe}

Stellenbosch University - Tygerberg Campus: Stellenbosch University Faculty of Medicine and Health Sciences

\section{Gerhard Walzl}

Stellenbosch University - Tygerberg Campus: Stellenbosch University Faculty of Medicine and Health Sciences

\section{Research article}

Keywords: Seroprevalence, antibodies, COVID-19, SARS-CoV-2, asymptomatic

Posted Date: December 29th, 2020

DOI: https://doi.org/10.21203/rs.3.rs-136543/v1

License: (c) (1) This work is licensed under a Creative Commons Attribution 4.0 International License. Read Full License 


\section{Abstract}

Background: South Africa has a high degree of inequality between population groups. The first wave of COVID-19 may have affected people in lower socioeconomic groups worse than the affluent. The SARSCoV-2 seroprevalence and the specificity of anti-SARS-CoV-2 antibody tests in South Africa is not known.

Methods: We tested 405 volunteers representing all socioeconomic strata from the workforce of a popular shopping and tourist complex in central Cape Town with the Abbott SARS-CoV-2 IgG assay. We assessed the association between antibody positivity and COVID-19 symptom status, medical history, and sociodemographic variables. We tested 137 serum samples from healthy controls collected in Cape Town prior to the COVID-19 pandemic, to confirm the specificity of the assay in the local population.

Results: Of the 405 volunteers tested one month after the first peak of the epidemic in Cape Town, 96(23.7\%) were SARS-CoV-2 IgG positive. Of those who tested positive, 46(47.9\%) reported no symptoms of COVID-19 in the previous 6 months. Seropositivity was significantly associated with living in informal housing, residing in a subdistrict with low income-per household, and having a low-earning occupation. The specificity of the assay was $98.54 \%(95 \% \mathrm{Cl} 94.82 \%-99.82 \%)$ in the pre-COVID controls.

Conclusions: There is a high background seroprevalence in Cape Town, particularly in people of lower socioeconomic status. Half of cases are asymptomatic, and therefore undiagnosed by local testing strategies. These results cannot be explained by low assay specificity.

\section{Introduction}

The first case of Coronavirus disease-2019 (COVID-19) in South Africa was documented on March 5th, 2020. Despite the early implementation of a stringent nationwide lockdown protocol, within 6 months South Africa (with a population of $\neg 60$ million people) had registered over 620000 cases of COVID-19, recording over 12000 new cases per day at the peak of the first wave $(1,2)$. The Western Cape Province of South Africa, a highly frequented tourist destination, suffered an earlier peak than the country's other provinces following several introductions of SARS-CoV-2 from Europe(3).

The City of Cape Town in the Western Cape Province is characterised by a startlingly high degree of inequality, with a Gini Index of 0.64 in 2014(4). Densely populated informal settlements, where residents live in shacks made of corrugated iron sheeting and are fully reliant on public transport, are found a few kilometres away from upscale leafy suburbs. During this epidemic, the inequalities between these areas have intensified. Many of the 'hotspots' of COVID-19 infections coincide with areas with lower per-capita income, and statistics show that the government-imposed containment measures were less effective there than in the more affluent suburbs(5).

Moreover, the number of reported cases does not reflect the true number of infections for several reasons. The national case definition relies on the identification of genetic material of SARS-CoV-2 on a respiratory specimen by reverse transcriptase-polymerase chain reaction (RT-PCR), a test with a sensitivity in the 
range of $80 \%(6)$. Local testing strategies were hampered by limited capacity and have mostly excluded people without symptoms(7). The proportion of asymptomatic cases is unknown, and estimates vary widely depending on the setting and the test format. Recently published seroprevalence studies confirm that a testing strategy which focuses purely on RT-PCR is likely to miss a large proportion of SARS-CoV-2 infections(8-10).

A recent sentinel surveillance study using residual blood samples from people attending routine antenatal and HIV care at public sector clinics in the Cape Town metropole, found SARS-CoV-2 seroprevalences ranging from $30.6-46.2 \%$ across all subdistricts(11). This suggests that certain areas and population groups in Cape Town have experienced a very pervasive first wave. However, the specificity of SARS-CoV2 antibody assays has not been assessed in this population, and the seroprevalence of people from higher income areas, who rarely access public sector healthcare, is not known. Here we report the results of a targeted workplace seroprevalence study in a popular shopping and tourist destination, which included people from all sociodemographic strata of Cape Town, as well as a control group from blood collected before November 2019.

\section{Methods}

Between 17 August and 4 September 2020 we recruited a diverse group of volunteers from all levels of employment of the Victoria and Alfred Waterfront (V\&A), which is South Africa's most-visited shopping and tourist destination in Cape Town, South Africa. This timepoint was approximately one month after the peak of the first wave of COVID-19 cases in this region and 6 months after the first documented case in the country. At the time of testing, South Africa was in the process of deescalating stringent lockdown measures which had already been in force for 5 months. During stringent lockdown most people did not attend work either part time or full time, or worked fewer days or hours, unless designated as an 'essential service' worker.

The workforce from which our volunteer sample was drawn included approximately 1200 workers either directly employed by the V\&A Waterfront, by one of the various museums and attractions on site, or by one of the companies acting as service providers. The volunteers were contacted and scheduled through their internal business communications structures. Volunteers were excluded if they had any symptoms of or known exposure to COVID-19 in the previous 10 days. Each participant attended a single visit in which they underwent informed consent counselling, and data on vitals and metrics, medical history, and COVID-19 exposure and symptoms were captured directly on to an electronic database.

Serum was collected from each participant, divided into aliquots and frozen at $-80^{\circ} \mathrm{C}$. Samples were analysed after a single freeze-thaw cycle with the Abbott SARS-CoV-2 IgG assay (Abbott Laboratories South Africa Pty Ltd) on the Architect i System (Abbott Laboratories, Illinois, USA), a chemiluminescent microparticle immunoassay (CMIA), for the qualitative detection of IgG antibodies to the SARS-CoV-2 nucleocapsid protein. An index of $\geq 1.4$ is considered positive according to the manufacturer's specifications. This assay has a reported sensitivity of $87.5-100 \%$, and a specificity of $99.63-100 \%(12-$ 
16). Using this $99 \%$ specificity, a power of $80 \%$, a significance level of 0.05 and a non-inferiority limit of $3 \%$, we calculated that a sample size of 137 pre-COVID controls would be sufficient to assert noninferiority of the Abbott SARS-CoV-2 IgG in the local population compared to the populations in which it has previously been tested and developed. We therefore tested 137 serum samples from asymptomatic controls from a low socioeconomic status local community, collected before November 2019.

Analysis was conducted using R. Continuous variables were expressed as median and interquartile range and tested for normality with the Shapiro-Wilk Test. Continuous variables with Gaussian distribution were analysed with a Student's t-test, and non-Gaussian variables with a Wilcoxon test. Categorical variables were compared using a Chi-squared test with continuity correction. Fisher's exact test was used to confirm significance, which was set at $p<0.05$. This study was approved by the Stellenbosch University Human Research Ethics Committee (reference N20/07/038-COVID-19).

\section{Results Of The Study Sample}

A total of 410 volunteers were screened. Four were excluded due to symptoms compatible with possible COVID-19 on the day, and one had an episode of syncope prior to blood draw. The details of the participants are presented in Table 1, stratified by their SARS-CoV-2 serology result. 
Table 1

SARS-CoV-2 seroprevalence, comorbidities, COVID-19 symptoms and exposure

\begin{tabular}{|c|c|c|c|c|}
\hline Demographic data & $\begin{array}{l}\text { Total }(n \\
=405)\end{array}$ & $\begin{array}{l}\text { Antibody Positive } \\
(n=96)\end{array}$ & $\begin{array}{l}\text { Antibody Negative } \\
(n=309)\end{array}$ & $\mathbf{p}$ \\
\hline Age (years) & $\begin{array}{l}38[18- \\
69]^{-}\end{array}$ & 36 [20-65] & 39 [18-69] & 0.108 \\
\hline Female gender $(n, \%)$ & $\begin{array}{l}217 \\
(53.6)\end{array}$ & $54(56.2)$ & $163(52.8)$ & 0.560 \\
\hline Comorbidities (n,\%) & 184 & $52(54.1)$ & $132(42.7)$ & 0.231 \\
\hline None & תרת & $15(15.6)$ & $42(13.6)$ & 0.873 \\
\hline Hypertension & $87(14.1)$ & $36(37.5)$ & $89(22.0)$ & 0.302 \\
\hline BP persistently $>140 / 90 \mathrm{mmHg}$ & $(30.9)$ & $10(10.4)$ & $12(3.9)$ & $0.040^{+}$ \\
\hline Diabetes & $22(5.4)$ & $9(9.4)$ & $19(6.2)$ & 0.375 \\
\hline HIV & $28(6.9)$ & $3(3.1)$ & $26(8.4)$ & 0.078 \\
\hline Asthma & $29(7.2)$ & $4(4.2)$ & $17(5.5)$ & 0.618 \\
\hline High Cholesterol & $21(5.2)$ & $1(1.0)$ & $5(1.6)$ & 1.000 \\
\hline Malignancy & $6(1.5)$ & $2(2.1)$ & $5(1.6)$ & 1.000 \\
\hline Heart disease & $7(1.7)$ & $2(2.1)$ & 5 & 1.000 \\
\hline Hypothyroidism & $7(1.7)$ & $1(1.0)$ & 8 & 0.463 \\
\hline Reflux & $9(2.2)$ & $0(0.0)$ & $4(1.3)$ & 0.577 \\
\hline Autoimmune disease & $4(1)$ & $0(0.0)$ & $3(1.0)$ & 1.000 \\
\hline Kidney disease & $3(0.7)$ & $8(8.3)$ & $12(3.9)$ & 0.120 \\
\hline Previous Tuberculosis & $30(7.4)$ & $25(26.0)$ & $127(41.1)$ & $0.006^{+}$ \\
\hline Ever smoker & $\begin{array}{l}152 \\
(37.5)\end{array}$ & & & \\
\hline \multicolumn{5}{|c|}{ Symptoms of COVID-19 in the previous 6 months $(n, \%)$} \\
\hline Any symptoms & $\begin{array}{l}180 \\
(44.4)\end{array}$ & $50(52.1)$ & $130(42.1)$ & NA \\
\hline
\end{tabular}




\begin{tabular}{|c|c|c|c|c|}
\hline Demographic data & $\begin{array}{l}\text { Total }(n \\
=405)\end{array}$ & $\begin{array}{l}\text { Antibody Positive } \\
(n=96)\end{array}$ & $\begin{array}{l}\text { Antibody Negative } \\
(\mathrm{n}=309)\end{array}$ & $\mathbf{p}$ \\
\hline Specific symptoms & $86(21.2)$ & $25(26.0)$ & $61(19.7)$ & 0.200 \\
\hline New cough & $67(16.5)$ & $28(29.2)$ & $39(12.6)$ & \multirow{2}{*}{$\begin{array}{l}< \\
0.001\end{array}$} \\
\hline Fever or chills & $59(14.6)$ & $21(21.9)$ & 38 (12.3) & \\
\hline Muscle aches & $33(8.1)$ & $11(11.5)$ & $22(7.1)$ & $0.030^{+}$ \\
\hline New dyspnoea & $76(18.8)$ & $16(16.7)$ & $60(19.4)$ & 0.200 \\
\hline Sore throat & $31(7.7)$ & $23(24.0)$ & $8(2.6)$ & 0.654 \\
\hline Loss of smell & $32(7.9)$ & $21(21.9)$ & $11(3.6)$ & 0.001 \\
\hline Loss of taste & $34(8.4)$ & $7(7.3)$ & $27(8.7)$ & \multirow{2}{*}{$\begin{array}{l}< \\
0.001\end{array}$} \\
\hline Diarrhoea & $11(2.7)$ & $1(1.0)$ & $10(3.2)$ & \\
\hline \multirow{2}{*}{ Nausea and/or vomiting } & & & & 0.833 \\
\hline & & & & 0.471 \\
\hline \multicolumn{5}{|l|}{ SARS-CoV-2 exposure $(n, \%)$} \\
\hline $\begin{array}{l}\text { Contact with a COVID-19 case in } \\
\text { the past } 6 \text { months }\end{array}$ & $70(17.3)$ & $14(14.6)$ & $56(18.1)$ & 0.296 \\
\hline Travel since December 2019 & $17(4.2)$ & $0(0.0)$ & $17(5.5)$ & 0.068 \\
\hline \& Outside South Africa & $87(21.5)$ & $17(17.7)$ & $70(22.7)$ & \\
\hline \&ithin South Africa & & & & \\
\hline
\end{tabular}

Participants' details stratified by their SARS-CoV-2 IgG antibody result, and factors associated with a positive antibody test. Age data are presented as median and range. ${ }^{+}$These $p$ values became nonsignificant after adjusting for the multiple testing effect via the Holm method. BP, blood pressure; HIV, Human Immunodeficiency Virus; COVID-19, coronavirus disease 2019.

The sample population consisted mostly of healthy adults, with a median age of 38 years, and median Body Mass Index (BMI) of 28 (range 16.5-57.6). Hypertension was the most common comorbidity, with $57(14.1 \%)$ of participants having a pre-existing diagnosis, and a further 125 participants had persistently elevated blood pressure readings on the day $(>140 / 90 \mathrm{mmHg})$. There were $19(4.7 \%)$ known diabetics, with 3 new diabetics diagnosed on site by point-of-care blood glucose and HbA1c testing. There were 28 (6.9\%) participants who self-declared as living with HIV, 24 of whom were on fixed-dose combination antiretroviral treatment. Other comorbidities are listed in Table 1.

Of the 405 participants included in the analysis, 96 (23.7\%) tested positive for SARS-CoV-2 IgG antibodies. On multivariate analysis, having diabetes and being an 'ever smoker' were associated with testing SARS-CoV-2 antibody positive, but became non-significant after correction for the multiple testing 
effect. Overall, $180 / 405$ (44.4\%) reported having had any symptoms of possible COVID-19 in the preceding 6 months. Of those who reported symptoms, 50/180 (27.8\%) tested antibody positive. Of the symptoms reported, only fever, muscle aches, loss of taste and loss of smell were significantly associated with a positive antibody test on multivariate analysis. There was no association between close contact with a known COVID-19 case and a positive antibody result. Only 17 participants travelled outside of South Africa after December 2019 and none tested positive for the SARS-CoV-2 antibody. There was no significant association between testing positive and travel within South Africa. Sixty-seven participants reported having undergone RT-PCR testing, of whom 15 (22.4\%) reportedly tested positive. Of these 15, ten tested positive for SARS-CoV-2 antibodies. In four of the five who reported testing RT-PCR positive but were SARS-CoV-2 antibody negative, the tests occurred more than 50 days apart (range 5087 days); the fifth was tested with RT-PCR 21 days before antibody testing. Of those who tested antibody positive and had had RT-PCR testing, 12 reported a negative PCR test result.

Three interlinked sociodemographic variables were identified which correlated to a positive antibody test: the participant's district of residence, their type of dwelling or housing, and their occupation (Table 2). 
Table 2

SARS-CoV-2 seroprevalence and sociodemographic indicators

\begin{tabular}{|lllll|}
\hline Sociodemographic indicators & Total & $\begin{array}{l}\text { Antibody } \\
\text { positive (n,\%) }\end{array}$ & $\begin{array}{l}\text { Antibody } \\
\text { negative (n,\%) }\end{array}$ & p \\
\hline Dwelling type: & 84 & $32(38.1)$ & $52(61.9)$ & $0.003^{+}$ \\
Informal housing & 321 & $64(19.9)$ & $257(80.1)$ & \\
Formal housing & & & & \\
Employment type: & 54 & $3(5.6)$ & $51(94.4)$ & 0.001 \\
Management & 150 & $19(12.7)$ & $131(87.3)$ & $<$ \\
Administration and Support & 50 & $16(32.0)$ & $34(68)$ & 0.001 \\
Parking and Security & 136 & $57(41.9)$ & $79(58.1)$ & 0.156 \\
Housekeeping services & 15 & $1(6.7)$ & $14(93.3)$ & $<$ \\
Other & 68 & $20(29.4)$ & $48(70.6)$ & 0.001 \\
'Essential services' designation* & & & & 0.211 \\
District of residence (\% of households with & 45 & $22(49)$ & $23(51)$ & 0.273 \\
income <\$10 per day): & 37 & $12(32.4)$ & $25(67.6)$ & 0.001 \\
Khayelitsha (49\%) & 34 & $11(32.4)$ & $23(67.6)$ & 0.226 \\
Mitchells Plain (42.3\%) & 41 & $11(26.8)$ & $30(73.2)$ & 0.292 \\
Klipfontein (38.8\%) & 106 & $22(20.8)$ & $84(79.2)$ & 0.700 \\
Tygerberg (28.4\%) & 63 & $5(7.9)$ & $58(92.1)$ & 0.426 \\
Southern (23.4\%) & 41 & $8(19.5)$ & $33(80.5)$ & 0.001 \\
Western (24.7\%) & 24 & $3(12.5)$ & $21(87.5)$ & 0.566 \\
Northern (21.1\%) & 5 & $1(20.0)$ & $4(80.0)$ & 0.222 \\
Eastern (13.5\%) & 9 & $1(11.1)$ & $8(88.9)$ & 1.00 \\
Cape Winelands, Overberg and West Coast & & & & 0.692 \\
Unknown & & & & \\
\hline
\end{tabular}

Sociodemographic indicators of participants stratified by their SARS-CoV-2 antibody result. District of Residence is listed in order of increasing income estimate, described by the percentage of households in that district with an income of less than $\$ 10$ per day. *Individuals who continued to attend work on site throughout all levels of lockdown. ${ }^{+}$This $p$ value became non-significant after adjusting for the multiple testing effect via the Holm method. 
Participants were stratified by health district of origin (in order to compare to reporting of locally registered cases). Districts were further defined by the percentage of households in each area with an income of less than $\$ 10$ per day, based on the most recent census data(17). The highest number of seropositive participants came from the Khayelitsha district (49\% of households with income $<\$ 10$ per day; SARS-CoV-2 seroprevalence $22 / 45,49 \%, p<0.001)$. Participants from the Southern district $(23.4 \%$ of households with income $<\$ 10$ per day) made up the largest group within the sample $(26.8 \%$ of participants) and had a seroprevalence of $22 / 106,20.8 \%(p=0.426)$. Participants from the Western district $(24.7 \%$ of households with income $<\$ 10$ per day) were significantly more likely to test negative and had a seroprevalence of $5 / 63(7.9 \%)(p<0.001)$. Figure 1 shows the relationship between the average income of the household in the district and the seroprevalence. Participants who lived in an informal dwelling were more likely to test positive, and those who live in a formal dwelling (house or flat) more likely to test negative $(p=0.001)$. When stratified by occupation, the largest proportion of the participants who tested positive arose from housekeeping services (cleaners), whereas the participants who worked in management were least likely to test SARS-CoV-2 antibody positive $(p<0.001)$ (Fig. 2).

On sub-analysis of the participants who tested antibody positive, no significant association was found between reporting symptoms of COVID-19 in the previous 6 months or not and any demographic variables, smoking status, BMI, or medical conditions. On analysing medication use (including ACEinhibitors, angiotensin receptor blockers, hydroxychloroquine, statins, oral and inhaled corticosteroids, antiretrovirals and multivitamin supplements) a significant association between statin use and symptomatic state was identified $(p=0.018)$, which became non-significant on correction for multiple testing effect $(p=0.546)$.

Results of the pre-COVID-19 controls

Of the 137 pre-COVID-19 serum samples analysed, two tested positive. Repeat testing of these two samples on the VIDAS SARS-CoV-2 IgG assay (bioMérieux, Midrand, South Africa) produced negative results for both. Assuming the absence of SARS-CoV-2 from the local population at the time of sample collection (before November 2019), this implies a specificity of $98.54 \%(95 \% \mathrm{Cl} 94.82 \%-99.82 \%, \mathrm{p}<0.05)$ for the Abbott SARS-CoV-2 IgG Assay in this population when using the manufacturer's recommended cut-off of $\geq 1.4$. Compared to the published specificity of $99.6 \%$ for this assay, this test demonstrated non-inferiority in our local population with a margin of $2.75 \%$ at a power of 0.8 and significance level of 0.05 .

\section{Discussion}

This study of a targeted workplace with high exposure to international visitors during the early phases of the first wave of the epidemic, demonstrates that people in lower socioeconomic groups of Cape Town have a higher seroprevalence of SARS-CoV-2 antibodies. Seroprevalence in participants' district of residence was strongly correlated with the percentage of people in the district with a low standardised measure of income. Moreover, participants in lower-earning occupations and those living in informal 
housing were more likely to test positive. Almost half of the participants from Khayelitsha - a partiallyinformal township in Cape Town afflicted by overcrowding and poverty - tested positive for SARS-CoV-2 antibodies.

Interestingly, there was no correlation between antibody positivity and so called 'essential services' designation. This implies that, despite working in an environment with high exposure to international tourists before lockdown, and the risks of continued use of public transport during lockdown, those infected were probably infected at home rather than the workplace. Rather, our data highlights the fact that some people are less able to comply with non-pharmacological interventions to prevent transmission than others because of their living circumstances. Similar inequalities have been documented in highincome countries: in New York City the number of COVID-19 cases detected by RT-PCR was significantly associated with multiple socioeconomic indicators, including population density, median household income, and dependent children(18). Another study compared the number of proven cases and deaths due to COVID-19 in each county of the USA to a poverty index. They found that early in the pandemic the counties with a higher poverty index had a higher number of cases, and throughout the pandemic these areas had higher number of deaths due to COVID-19(19). In Leicester, UK, larger household size and belonging to an ethnic minority were both associated with a higher likelihood of testing SARS-CoV-2 RTPCR positive(20). Jay and colleagues used smartphone tracking data in the USA to demonstrate that the 'stay at home' orders were only associated with small increases in staying home in low-income neighbourhoods compared to high-income neighbourhoods (21). Our participants from low-income districts face multiple physical barriers to the social distancing and stay-at-home orders, and these may explain the higher seroprevalence in these areas.

The overall seroprevalence in the current study of $23.7 \%$ after the first wave, is higher than in countries with high average income, low inequality and an ability to shelter their population, such as Germany and Ireland(22). However, our result is similar to a sample from a pilot study in Niger State, Nigeria (Gini index 0.35 ) which reported a seroprevalence of $25.4 \%$ independent of rural or urban residence(23), and to the studies which reported higher seroprevalences in more densely populated areas within countries. For example, the overall seroprevalence of a convenience sample from New York State in the USA was 12.5\%, but in New York City itself, where population density and inequality between neighbourhoods is higher, the estimated cumulative incidence of COVID-19 cases was $22.7 \%$.(24). A Spanish seroprevalence survey found an overall seroprevalence of only $4.7 \%-5 \%$ in May of 2020, which increased to $14.4 \%$ in the more densely populated central provinces(9).

In our study almost half of all positive participants were completely asymptomatic of COVID-19 in the previous 6 months, suggesting that socioeconomic status of participants was not associated with higher severity of disease. Even though the prevalence of uncontrolled or undiagnosed comorbidities which may predispose to severe COVID-19 is expected to be higher in areas of low socioeconomic status, this group may not be part of the active workforce and so were not included in our sample. The seropositiveasymptomatic proportion in our sample is similar to that in other studies. The Spanish study reported that between $28.5 \%$ and $32.7 \%$ of all who tested positive had not had symptomatic COVID-19(9). In an 
Icelandic study 41 (3.3\%) of 1244 people in quarantine for SARS-CoV-2 exposure tested antibody positive, of whom 24 (58.5\%) reported no symptoms(10). The high proportion of asymptomatic infections contributes to the case detection and reporting gap, along with patients with mild symptoms often not presenting to healthcare at all, and the effect of the highly targeted testing strategies employed in South Africa due to limited testing capacity.

The presence of SARS-CoV-2 antibodies in serum has not yet been definitively correlated with long term immune memory. However, evidence of a high baseline seroprevalence (at least in some regions) is encouraging as it could mean that communities less shielded from infection pressure for socioeconomic reasons may at least be spared an overwhelming 'second wave', barring any significant mutations to the virus which impact the efficacy of neutralising antibodies.

Our study found an unexpected association between reporting symptoms in keeping with COVID-19 in the previous 6 months and the use of statins, which have previously been associated with a reduced severity of COVID-19(25). It's possible that our sample method selected out those who suffered severe COVID-19, and that statins should rather be considered marker of comorbidity (and therefore higher risk for symptomatic COVID-19) in this population.

In our local pre-COVID-19 control group the specificity of the assay used in our study was non-inferior to the reported specificity, supporting the reliability of our findings. A limitation of our study is the sampling method, which resulted in unequal numbers from each district and occupational group. Our sample excluded those who are not part of the labour force, children, and the elderly. Our offer of serologic testing for SARS-CoV-2 before it was freely available may have introduced bias by selecting people who had a strong suspicion of having been infected. Nonetheless, we recruited a high proportion $(405,34 \%)$ of the potential pool of 1200 volunteers and our sample included participants from a broad range of sociodemographic backgrounds, less than half of whom ever experienced symptoms of possible COVID19 and very few of whom had contact with a known case. Retrospectively, it was felt that the participant's use of public versus private transport and the number of people in their household would have been useful sociodemographic indicators to analyse, but this data was not collected. Lastly, we were unable to confirm the RT-PCR results of those participants who reported having the test, which precluded further meaningful analysis of this data.

\section{Conclusion}

In this study we have shown that the Abbott SARS-CoV-2 IgG assay is highly specific in our population. We found a higher seroprevalence than most other published reports, but a similar proportion of asymptomatic infections. We have also demonstrated that the local epidemic has affected people of lower socioeconomic status worse than the affluent, likely due to an inability of either the local government structures or the individual to shield against infection pressure, both physically and financially. Future infection containment measures should take socioeconomic factors into consideration as those applied during this pandemic were less effective in vulnerable populations. 


\section{Declarations}

\section{Ethics approval}

This study was approved by the Stellenbosch University Human Research Ethics Committee, reference number N20/07/038-COVID-19.

\section{Consent to participate}

Participation in this study was entirely voluntary, and every participant underwent full informed consent counselling and signed an informed consent form prior to taking part.

\section{Consent for publication}

Not applicable.

\section{Availability of data and materials}

The datasets used analysed during the current study are available are captured in an online RedCap database and available from the corresponding author on reasonable request.

\section{Competing interests}

The authors declare that they have no competing interests.

\section{Funding}

This study was co-funded by the Stellenbosch University Immunology Research Group and the Victoria \& Alfred Waterfront Pty, Ltd. The Victoria \& Alfred Waterfront Pty, Ltd had no role in the study design, data analysis and interpretation, or in the writing of the manuscript. Personnel from the Victoria \& Alfred Waterfront Pty, Ltd assisted the research team in recruiting potential volunteers through their internal communication networks, but were not involved in the informed consent process.

\section{Authors' contributions}

JS, TC, NdP, NC, LK, AGL, HR, WP, STM, GW contributed to the design of the study and interpretation of results; JS, TC, DS, CC, NM, VL, CM, AH, MF, STM, KS were integral to the collection of the data and execution of the study; MM and GT performed the analysis; JS drafted the manuscript, all authors read and approved the final manuscript.

\section{Acknowledgements}

We extend our thanks to the V\&A Waterfront for proposing and funding the project, and to Mr Andre Theys, his administrative team, and the V\&A community advisory board for the warm welcome and continuous support throughout its execution. 


\section{References}

1. National Institute for Communicable Diseases South Africa. https://www.nicd.ac.za/wpcontent/uploads/2020/09/COVID-19-National-Daily-Report_Public_01092020.pdf Accessed 26 October 2020.

2. University of Oxford Coronavirus government response tracker.

https://www.bsg.ox.ac.uk/research/research-projects/coronavirus-government-response-tracker. Accessed 26 October 2020.

3. Wilkinson E, Tegally H, de Oliveira T, Delaney K, Kleinhans B, Preiser W, et al. Molecular epidemiology of SARS-CoV-2 in Cape Town, South Africa. Nextstrain. 2020. https://nextstrain.org/groups/ngssa/narratives/COVID19-WC-2020.07.13 Accessed 6 November 2020.

4. State of Cape Town Report. 2016.

http://resource.capetown.gov.za/documentcentre/Documents/City research reports and review/State of Cape Town 2018.pdf Accessed 3 November 2020.

5. Western Cape Government COVID-19 dashboard. https://coronavirus.westerncape.gov.za/covid-19dashboard \%0A12\%0A. Cited 29 September 2020.

6. Kucirka LM, Lauer SA, Laeyendecker O, Boon D, Lessler J. Variation in False-Negative Rate of Reverse Transcriptase Polymerase Chain Reaction-Based SARS-CoV-2 Tests by Time Since Exposure. Ann Intern Med. 2020;173(4):262-7.

7. Porter JD, Mash R, Preiser W. Turnaround times - the Achilles' heel of community screening and testing in Cape Town, South Africa: A short report. African J Prim Heal Care Fam Med. 2020;12(1). Available from: https://phcfm.org/index.php/phcfm/article/view/2624.

8. Stringhini S, Wisniak A, Piumatti G, Azman AS, Lauer SA, Baysson H, et al. Seroprevalence of antiSARS-CoV-2 IgG antibodies in Geneva, Switzerland (SEROCoV-POP): a population-based study. Lancet. 2020;396(10247):313-9.

9. Pollán M, Pérez-Gómez B, Pastor-Barriuso R, Oteo J, Hernán MA, Pérez-Olmeda M, et al. Prevalence of SARS-CoV-2 in Spain (ENE-COVID): a nationwide, population-based seroepidemiological study. Lancet. 2020;396(10250):535-44.

10. Gudbjartsson DF, Norddahl GL, Melsted P, Gunnarsdottir K, Holm H, Eythorsson E, et al. Humoral Immune Response to SARS-CoV-2 in Iceland. N Engl J Med. 2020;383(18):1724-34.

11. National Institute for Communicable Diseases South Africa. SARS-CoV-2 seroprevalence in the Cape Town Metropolitan Subdistricts after the peak of infections. COVID-19 Special Public Health Surveillance Bulletin 18(Supplementary issue 5). https://www.nicd.ac.za/wpcontent/uploads/2020/09/COVID-19-Special-Public-Health-Surveillance-Bulletin_Issue-5.pdf.

12. Bryan A, Pepper G, Wener MH, Fink SL, Morishima C, Chaudhary A, et al. Performance Characteristics of the Abbott Architect SARS-CoV-2 IgG Assay and Seroprevalence in Boise, Idaho. J Clin Microbiol. 2020;58(8):e00941-20. 
13. Hamilton F, Muir P, Attwood M, Vipond ANB, Hopes R, Moran E, et al. Kinetics and performance of the Abbott architect SARS-CoV-2 IgG antibody assay. J Infect. 2020;(S0163-4453(20)30510-7).

14. National SARS-CoV-2 Serology Assay Evaluation Group. Performance characteristics of five immunoassays for SARS-CoV-2: a head-to-head benchmark comparison. Lancet Infect Dis. 2020; (S1473-3099(20)30634-4).

15. Paiva KJ, Grisson RD, Chan PA, Huard RC, Caliendo AM, Lonks JR, et al. Validation and performance comparison of three SARS-CoV-2 antibody assays. J Med Virol. 2020.

16. Theel ES, Harring J, Hilgart H, Granger D. Performance Characteristics of Four High-Throughput Immunoassays for Detection of IgG Antibodies against SARS-CoV-2. J Clin Microbiol. 2020;58(8).

17. Cape Town census and population statistics: Health District Profiles. 2011 [https://www.capetown.gov.za/Family and home/education-and-research-materials/data-statisticsand-research/cape-town-census. Accessed 12 November 2020.

18. Whittle RS, Diaz-Artiles A. An ecological study of socioeconomic predictors in detection of COVID-19 cases across neighborhoods in New York City. BMC Med. 2020;18(1):271. doi:10.1186/s12916-02001731-6.

19. Finch WH, Hernández Finch ME. Poverty and Covid-19: Rates of Incidence and Deaths in the United States During the First 10 Weeks of the Pandemic. Front Sociol. 2020;5:47. https://www.frontiersin.org/article/10.3389/fsoc.2020.00047.

20. Martin CA, Jenkins DR, Minhas JS, Gray LJ, Tang J, Williams C, et al. Socio-demographic heterogeneity in the prevalence of COVID-19 during lockdown is associated with ethnicity and household size: Results from an observational cohort study. EClinicalMedicine. 2020;25:100466.

21. Jay J, Bor J, Nsoesie EO, Lipson SK, Jones DK, Galea S, et al. Neighbourhood income and physical distancing during the COVID-19 pandemic in the United States. Nat Hum Behav. 2020. doi:10.1038/s41562-020-00998-2.

22. Arora RK, Joseph A, Van Wyk J, Rocco S, Atmaja A, May E, et al. SeroTracker: a global SARS-CoV-2 seroprevalence dashboard. Lancet Infect Dis. 2020. doi:10.1016/S1473-3099(20)30631-9.

23. Majiya H, Aliyu-Paiko M, Balogu VT, Musa DA, Salihu IM, Kawu AA, et al. Seroprevalence of COVID-19 in Niger State. medRxiv. 2020;2020.08.04.20168112. http://medrxiv.org/content/early/2020/08/05/2020.08.04.20168112.abstract.

24. Rosenberg ES, Tesoriero JM, Rosenthal EM, Chung R, Barranco MA, Styer LM, et al. Cumulative incidence and diagnosis of SARS-CoV-2 infection in New York. Ann Epidemiol. 2020;48:23-9.e4.

25. Tan WYT, Young BE, Lye DC, Chew DEK, Dalan R. Statin use is associated with lower disease severity in COVID-19 infection. Sci Rep. 2020;10(1):17458. doi:10.1038/s41598-020-74492-0.

\section{Figures}




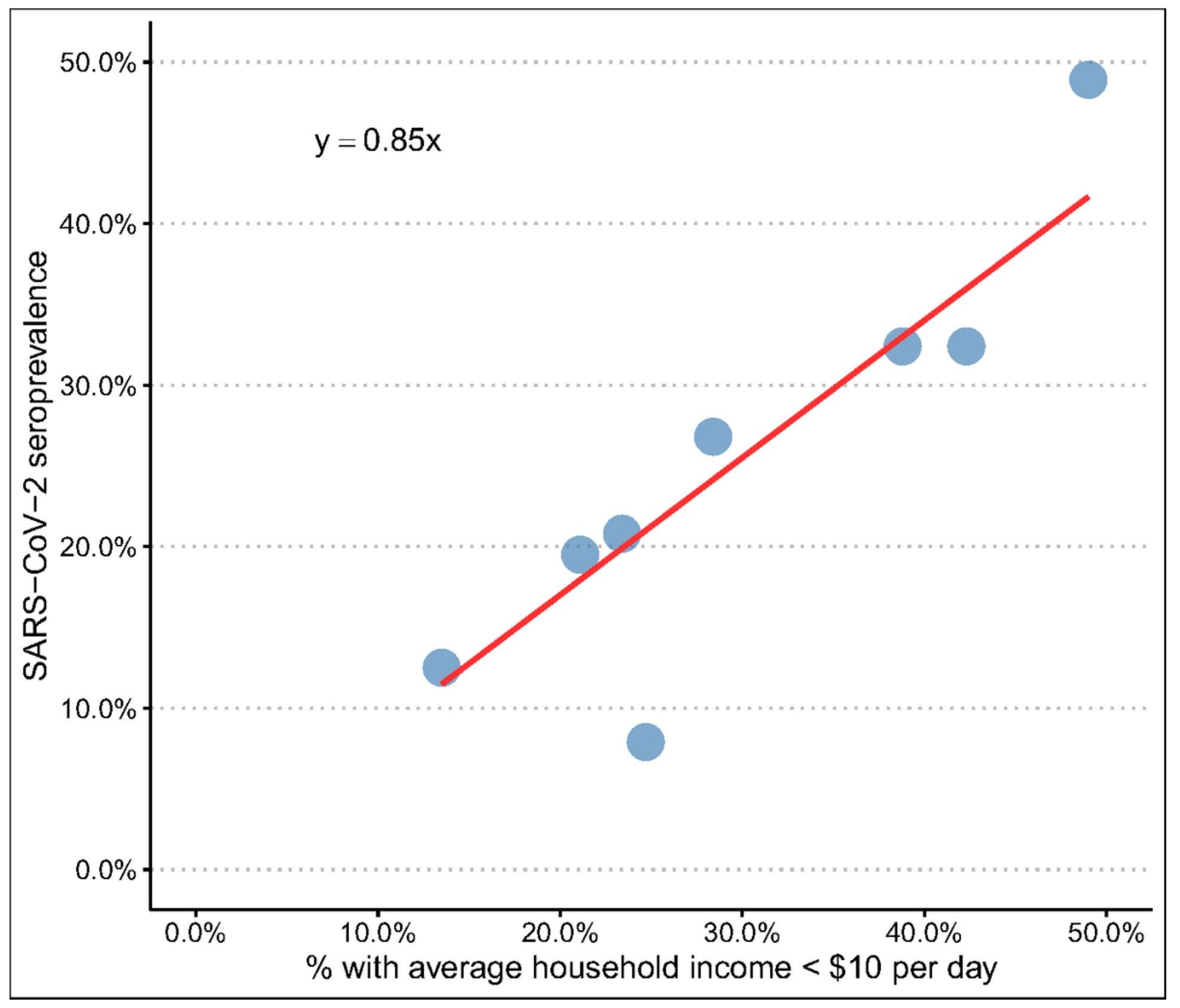

Figure 1

The correlation between the income and the SARS-CoV-2 seroprevalence within the subdistrict of residence of the study population. Income is denoted by the proportion of households with a daily income of $<\$ 10$, with an increasing value implying a lower socioeconomic status. 


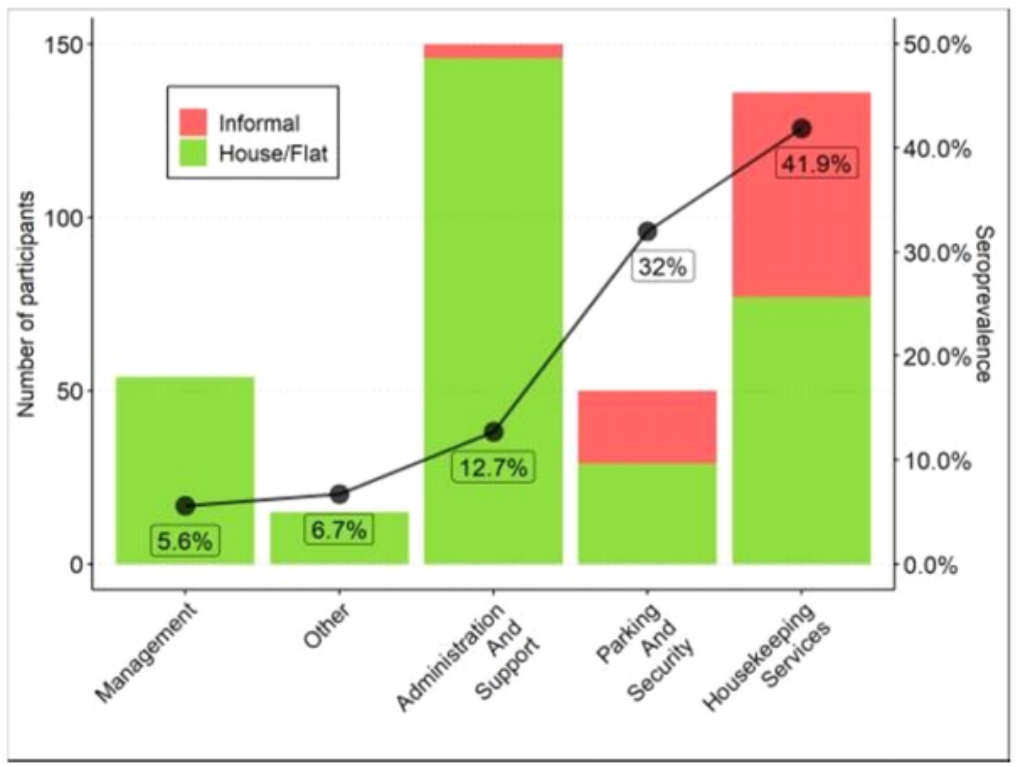

Figure 2

The association between participants' occupations, type of dwelling and SARS-CoV-2 seroprevalence. There is a higher seroprevalence in lower-earning occupation groups with a higher proportion of participants living in informal housing. 